\title{
Unusual echocardiographic appearance of intracardiac thrombi in a patient with endomyocardial fibrosis
}

Sir,

We were interested to read the report by Wiseman et al (1986;56:179-81) describing a patient with intracardiac thrombi which had echolucent centres.

We recently encountered a similar echocardiographic appearance in a 72 year old woman admitted with cardiac failure. Cross sectional echocardiography showed biventricular dilatation with uniformly reduced ventricular function that was

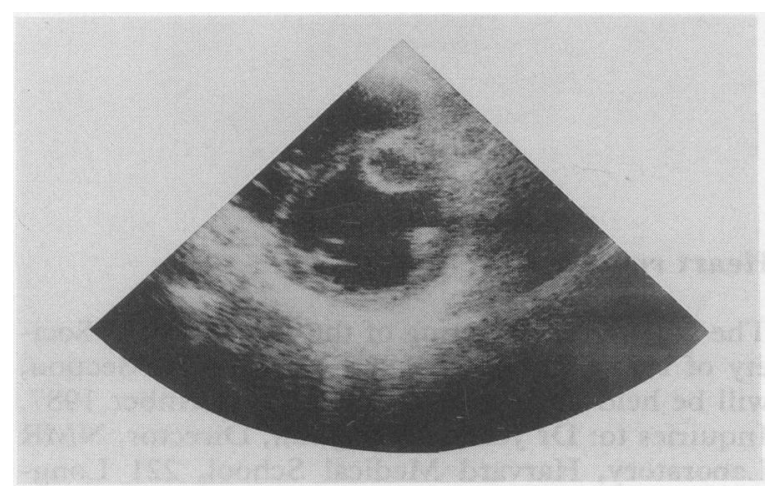

Figure Echocardiogram in the parasternal short axis view at the level of the papillary muscles showing the mass with an echolucent centre. compatible with a congestive cardiomyopathy. spherical mass with a thin echogenic perimeter and o large echolucent centre was seen to be attached tọ the anteroseptal wall of the left ventricle just below the level of the papillary muscles. Although cardiae tumours can have small echolucent areas, we felt that the position and the clinical setting of this lesion made ventricular thrombus with liquefaction at it centre the most likely diagnosis. Despite antiê coagulation the patient sustained a left sided hemiplegia ten days after admission and repease echocardiography showed the lesion to be smaller and uniformly echodense. We believe that the echo cardiographic findings were the result of a thrombus with a liquefied centre which subsequently becam $\bar{S}$ partly embolised. The histology in the case reported by Wiseman et al lends support to our presumptiv diagnosis.

M Buchalter, J P Bourke, $S$ Tansuphaswadikul, Department of Cardiology, Freeman Hospital, Newcastle upon Tyne, NE7 7DN. 